\title{
Ueber das Fehlen einer Querschichtung in den Kernen der menschlichen Stäbchensehzellen'1).
}

\author{
Von
}

W. Flemming,

Prof. in Kiel.

Die Querschichtung der „äusseren Körner" ist bekanntlich von $\mathrm{Henle}(1864)^{2}$ ) entdeckt, und weiter vielfach, so von Rit ter ${ }^{3}$ ), Krause ${ }^{4}$ ), Schwalbe ${ }^{5}$ ), Dennissenko ${ }^{6}$ ) und mir selbst ${ }^{7}$ ) untersucht worden. Henle, Ritter und $\mathrm{Kr}$ a u se allein sind es, so viel ich finden kann, die in Bezug darauf über positive Befunde beim Menschen berichten, von denen alsbald die Rede sein soll.

Ich hatte am eben citirten Orte (1882) den Bau der Stäbchenkerne bei der Katze, dem Kaninchen und Meerschwein geprüft. Ich fand die Querschichtung bei allen diesen Thieren, sowohl am frischen zerzupften Object, als nach Fixirung mit verschiedenen Reagentien und Färbung ganz deutlich ausgesprochen, besonders augenfällig bei der Katze. Die Angabe W. Krause's, dass die Schichten wie kleine Convex- oder Concavlinsen mit ganz regelmässig gestalteten Flächen geformt seien, fand ich nicht

1) Vorläufig mitgetheilt im physiolog. Verein zu Kiel, Sitzung vom 12. Juli 1897, 3. Münchener med. Wochenschrift Nr. 41, 1897, pag. 1153.

2) Göttingische geel. Anzeigen 1864, 1, pag. 119, und ebenda 1864, 2, pag. 305; ferner in Henle's Handbuch d. Anat. Eingeweidelehre 1873.

3) Arch. f. Opthalmologie, Bd. 11, 1865, p. 83.

4) Handbuch der menschl. Anat., allg. Anat. p. 161, Der (bei Schwalbe, s. u., citirte) Aufsatz Kra use's: Die membraua fenestrata der Retina, Leipz. 1868, war mir hier nicht zugänglich.

5) Schwalbe, Mikrosk. Anat. der Netzhaut, des Sehnerven und des Glaskörpers, in: Handb. d. Ophthalmol. v. Graefe u. Saemisch, Bd. 1, p. 420-421. - Derselbe : Lehrbuch der Anatomie der Sinnesorgane, 1886, p. 106.

6) Arch. f. miks. Anat. Bd. 19.

7) Zellsubstanz, Kern und Zelltheilung 1882, p. 114-121. 
Ueber das Fehlen einer Querschichtung in den Kernen etc. 705

bestätigt; die Flächen sind sehr vielfach uneben und es gehen sogar hie und da brückenartige kleine Fortsätze von einer zur anderen Schicht hinüber, wie dort beschrieben ist. Die Querschichten sind stark färbbar; es handelt sich eben einfach darum, dass die chromatinhaltige Substanz im Kern in zwei bis drei dichte Schichten zusammengedrängt liegt. Die Meinung Dennissenko's, nach der die Querstreifen nicht ganz durch die Kerne hindurchreichen und sogar nur einseitig liegen sollen, trifft nach meinen Untersuchungungen nicht zu. Für Näheres hierüber verweise ich auf meine dort gegebenen Abbildungen und den Text. Die Existenz von $\mathrm{Nu}$ cleole $\mathrm{n}$, die ich damals gleichfalls beschrieb, ist ganz sicher und ich habe sie seitdem mehrfach, unter Anwendung von Osmiumsäure, bestätigen können. Sie ist übrigens schon früher von $W . K \mathrm{ra} \mathrm{u} \mathrm{se}^{1}$ ) angegeben.

Die Vermuthung M a x S c u ltz e's²), dass die Querschichtung der Stäbchenkerne lediglich eine Leichenerscheinung sei, ist nach meiner Untersuchung an ganz frischen Retinen gewiss nicht aufrecht $\mathrm{zu}$ erhalten.

Schon Max Schultze'hatte an diesen Stellen gesagt und Schwalbe ${ }^{3}$ ) hatte es bestätigt, dass die Querschichtnng auf die Stäbchenkerne der Säugethiere beschränkt, bei Amphibien, Reptilien, Vögeln nichts davon zu sehen sei. Ich habe es (a. a. 0.) bei Amphibien ganz ebenso gefunden, und mir ist auch seitdem keine Angabe bekannt geworden, die dem widerspräche. Uebrigens hat schon Ritter (a.a.0.p. 98) nach Untersuchung bei verschiedenen Vögeln und vielen Fischen es wahrscheinlich gefunden, dass die Erscheinung nur bei Säugethieren vorkommt.

Der menschlichen Retina aber schreibt Schwalbe an der erwähnten Stelle die Querschichtung zu und ebenso thun es andere neuere Schriftsteller. Da aber hierbei keine eigenen Befunde angeführt werden, so möchte ich annehmen, dass diese Autoren sich dabei auf diejenigen von Henle, Ritter und $\mathrm{Kr}$ a u s e bezogen und verlassen haben ${ }^{*}$ ). paraten.

1) Allgem. Anatomie 1876, p. 161; gleichfalls nach Osmiumprä-

2) Die Retina. Stricker's Handbuch der Gewebelehre, p. 955; vergl. auch Arch. f. mikr. Anat. Bd. 2, p. 219.

3) Mikrosk. Anatomie der Netzhaut etc. in: Graefe u. Sa emisch' Handb. d. Ophthalm.; p. 421.

4) Denn die Stelle bei Schwalbe (Mikrosk. Anat. des Seh- 
Ich habe seit jenen meinen Arbeiten gesucht, mir für diesen und andere $Z$ wecke ganz frische menschliche Bulbi zu verschaffen und die Retina sofort zu fixiren, und habe durch die Güte meines Collegen Völckers, sowie von anderen Seiten nach und nach eine ziemliche Anzahl solcher erhalten. Meistens habe ich die Retina so fixirt, dass am hinteren Theil des Bulbus ein Scleragürtel möglichst rasch abpräparirt wurde, was bei einiger Uebung in wenigen Minuten gelingt, und dann das ganze Auge in Chromosmiumessigsäure, Osmiumsäure, Alkohol oder $1 / 4$ procentige Chromsäure geworfen wurde. Von ganz frischen Augen habe ich in solcher Weise drei mit Chromosmiumessigsäture, zwei mit Osmiumsäure, drei mit Alkohol und eins mit Chromsäure fixirt, ausserdem mehrere längere Zeit post mortem. Bei all diesen Augen habe ich nun, nach Einbettung, Schnitt und Färbung in verschiedener Weise ${ }^{1}$ ), niemals eine Spur von Querschichtung an den Stäbchenzellenkernen der Retina zu Gesicht bekommen. An den Präparaten aus Osmiumgemisch zeigen diese Kerne scharfausgesprochene, gut färbbare chromatische Gerüste. Die Osmiúmsäurepräparate gewähren entweder, nur etwas undeutlicher and verwaschener, dasselbe Bild, oder die Stäbchenkerne sehen daran homogen aus und zeigen sehr blasse Nucleolen, was also der gewöhnlichen Osmiumsäurewirkung auf Zellkerne entspricht ${ }^{2}$ ). An den Alkohol- und Chromsäurepräparaten sind die leicht geschrumpften Stäbchenkerne, bei Hämatein- oder Carminfärbung, entweder gleichfalls netzig, oder sehen fast homogen aus; von irgendwelcher Schichtung aber ist auch in ihnen durchaus nichts $z a$ entdecken.

Dies muss um so mehr Wunder nehmen, als nach meinen bisherigen Erfahrungen die gleichen Behandlungen bei anderen Thieren die schönsten Bilder von Querschichtung der Stäbchenkerne

nerven etc. p. 420), nach welcher er die Querstreifung an ganz frischen Präparaten deutlich wahrgenommen hat, kann sich wohl auf Thieraugen beziehen.

1) Meistens Stückfärbung mit Hämatein oder Alauncarmin, $\mathrm{Pa}$ raffin; ausserdem Saffranin- und Carminfärbungen an Celloidinpräparaten.

2) Bei diesen Kernen giebt die Osmiumsäure nur dann, wenn sie kurz oder mehr verdünnt wirkt, das Resultat, dass der Kern klar aussieht und Nucleolen zeigt (vergl. oben); bei stärkerer oder längerer Wirkung wird er, wenigstens häufig, netzig. 
Ueber das Fehlen einer Querschichtung in den Kernen etc. 707

liefert: bei Schnitten von Katzenretinen z. B., die mit Chromosmiumessigsäure fixirt und mit Safranin gefärbt sind, ist solche schon bei mittelstarker Vergrösserung auf den ersten Blick deutlich.

Es ist mir in Bezug auf dieses Fehlen der Querschichtung. beim Menschen nichts Anderes bekannt, als eine kurze Bemerkung Dogiel's rom Jahre 1884 ${ }^{1}$ ), welche lautet: „An Präparaten (der menschlichen Retina), die mit $\mathrm{M}$ üll e r'scher Lösung, Alkohol, Chrom- oder Osmiumsüure behandelt wurden, erscheinen die Kerne (der Stäbchensehzellen) stets granulirt, ohne jede Spur von Querstreifen, wie sie Henle zuerst beschrieb. ${ }^{\text {" }}$ Ich mag jedoch vielleicht in der grossen Literatur der Retina andere hierher gehörige Angaben übersehen haben.

Es konnte nun noch die Frage sein, ob vielleicht beim Menschen der Querbau der Kerne zwar intra vitam vorbanden ist, aber hier post mortem sich besonders leicht verwischt. Dieser Gedanke kann dadurch angeregt werden, dass die vorher erwähnten, älteren Literaturangaben über Querschichtung der menschlichen Stäbchenkerne sich fast durchweg auf die frische Retina beziehen ${ }^{2}$ ).

Ich habe also, obwohl ich über die oben mitgetheilten Erfahrungen schon seit längeren Jahren verfüge, uiber diese Sache

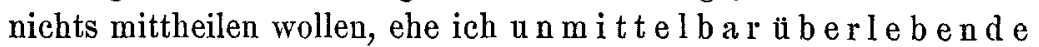
menschliche Retinen darauf untersucht hatte; so bedauerlich es war, die Bulbi bloss für diesen Zweck zu opfern. Im letzten Juli erhielt ich von Herrn Collegen Völkers ein Auge eines 10 jährigen Kindes, wegen retrobulbären Tumors enucleirt, der Bulbus intact, $1 / 4$ Stunde nach der Operation. Der Bulbus wurde sofort angeschnitten und ein Stück Retina, in Humor vitreus rasch zerzupft, betrachtet. Das Gewebe war so frisch, dass in der Retina anfangs die Structurtheile kaum zu erkennen waren und erst allmählich deutlicher wurden. In den Stäbchenkernen traten zuerst, sehr blass, aber erkennbar, die Nucleoli auf, dann

1) Ueber die Retina des Menschen. Internationale Monatsschr. f. Anat. und Histol., 1884, Bd. 1, p. 172.

2) Henle, am erst cit. Orte p. 121 ; Ritter, a. a. 0. p. 91 u. 93. Letzterer sagt dort, dass die Querstreifen einige Stunden nach drm Tode verschwinden, sich aber in Kalibichromat einige Tage, in Alkohol sehr gut und lange erhalten. 
trübte sich rasch der ganze Kern mit einer durchgehenden matten Granulirung, die obne Zweifel den durch Reagentien und Färbung deutlich zu machenden Kerngerüsten entsprach; irgend ein Uebergangsstadium, welches auch nur Spuren einer Querschichtung gezeigt hätte, war nicht zu sehen.

Einen anderen Bulbus erhielt ich im Norember d. J. etwa $1 / 2$ Stunde post mortem; er gehörte einem 31 jührigen Manne an. An dem sofort entnommenen Retina-Zupfpräparat zeigte sich das Gleiche wie im vorigen Fall : nachdem etwa $1 / 2$ Minute nach Eindeckung des Präparats die Stäbchenkerne kaum zu erkennen waren, wurden sie scheinbar granulirt, d. h. netzig; nur das kurze Uebergangsstadium, in dem man im vorigen Fall bloss die $\mathrm{Nu}$ cleolen sah, konnte ich hier nicht beobachten. Von einer Querschichtung war auch hier durchaus nichts zu erkennen ${ }^{\mathbf{1}}$ ).

Hiernach glaube ich mich berechtigt, meine Ergebnisse nitzutheilen. Sie stehen in Widerspruch mit den Angaben Ritter's und denen $\mathrm{Kr}$ a u se's; etwas weniger mit denen des Entdeckers dieser Structur, Henle's, denn dieser äussert sich darüber in Bezug auf die menschliche Retina sehr vorsichtig. Er scheint zwar nicht daran zu zweifeln, dass die Querschichtung auch dieser zukommt ${ }^{2}$ ), er sagt aber an einer früheren Stelle ${ }^{3}$ ), dass er an der grossen Mehrzahl der menschlichen Stäbchenkerne (einige Stunden post mortem) nichts davon habe sehen können, nur hätten sich allerdings "einzelne gefunden, die in gewissen Lagen dieselben Querstreifen zeigten, wie die Aussenkörner der Säugethiere" ${ }^{\prime}$, und er bezieht sich an der oben citirten Stelle in der Eingeweidelehre, welche die Querschichtung schildert, so viel ich sehen kann, auch nicht speciell auf eigene Beobachtungen an der menschlischen Retina.

Die. Mittheilungen Ritter's und Krause's dagegen lauten allerdings ganz positiv. Ersterer sagt, dass er die Querschichtung genauer beim Lamm, beim Kalb und beim Menschen

1) Die Beobachtung wurde im ersten Falle mit einer sehr guten Wasserimmersion 9 von $\mathrm{Hartnack}$, im zweiten mit Zeiss Apochrom. $2 \mathrm{~mm} \mathrm{1,40}$ gemacht.

2) Siehe die Darstellung in Henle's Eingeweidelehre, 1873, p. $676-678$.

3) S. am zweitcit. Ort im Anfang dieses Aufsatzes, p. 307. 
untersucht, und unter anderen auch am Auge eines Kindes deutlich gefunden habe, das einige Tage in Alkohol gelegen hatte. Einige Zeit nach dem Tode soll nach ihm die Querschichtung. verschwinden; in verdünnter Chromkalilösung sich einige Tage, in Alkohol, besonders halbverdünntem, gut und lange erhalten. Ritter ist der Meinung, dass bei einer Erhärtung, die sich zu guten Querschnitten der Retina eignet, die Querschichtung nicht zu sehen sei, dagegen bei einer geringeren Erhärtung, welche keine Schnitte gestatte, sich selur gut prüsentire. Ersteres ist aber offenbar nicht richtig, denn ich habe ja oben gesagt, dass sie bei Thierretinen, die in ganz frischem Zustand gut fixirt sind, vortrefflich zu sehen ist. - Die Arbeit Ritter's ist schon älteren Datums (1865), und es könnte gegen sie etwas misstrauisch machen, dass der Verfasser darin auf das Entschiedenste behauptet, die „üusseren Körner" seien keine Zellkerne. Doch waren die Mikroskope ja damals schon so leistungsfähig, dass eine vollständige Täuschnng in Bezug auf den Bau der menschlichen Sehzellenkerne schwer begreiflich erscheinen würde.

In W. Kra use's nAllgemeiner Anatomie ${ }^{*}$ ist auf S. 191, wo die Querschichtung beschrieben wird, zwar nicht speciell von der menschlichen Netzhaut die Rede, man muss aber annehmen, dass der Verfasser die Erscheinung auch dieser zuschreibt, da er kurz zuvor (p. 156) in Fig. A gre ein geschichtetes Stäbchenkorn vom Menschen, $1 / 4$ Stunde p. mortem in Glaskörperflüssigkeit isolirt, und daneben einen menschlichen Zapfen mit geschichtetem Kern zeichnet. Kra us e schrieb nämlich auch den Zapfenkernen die Querschichtung zu, was aber von Niemand bestätigt worden ist.

Beiden Autoren gegenüber kann ich also in den menschlichen Stäbchenkernen weder frisch, noch nach verschiedenartiger Conservirung etwas von Querschichtung finden. Es ist ohne Zweifel sehr merkwürdig, dass sie hier zu fehlen scheint, da sie in den gleichen Kernen bei so vielen Säugethieren ohne Zweifel $z u$ finden ist. Freilich sind auch noch lange nicht alle Sängethiere darauf untersucht ${ }^{1}$ ). Dass sie für das Sehen über-

1) Gefunden ist die Querschichtung meines Wissens: bei Katze, Kaninchen, Meerschwein, Wiederkiunern und Pford. 
710 W. Flem m in g: Ueber das Fehlen einer Querschichtung etc.

haupt keine wesentlichere Bedentung haben kann, geht wohl schon aus ilrem Fehlen bei den anderen Wirbelthierklassen hervor.

Wenn ich im Obigen meine negativen Befunde festgestellt habe, bin ich doch weit von der Behauptung entfernt, dass etwas, weil ich es nicht habe finden können, nicbt existiren köunte. Sollte irgend Jemand meiner Herren Fachgenossen Präparate besitzen, die an menschlichen Stäbchewzellenkernen etwas von Querschichtung zeigen, so möchte ich anregen, dass dieselben auf dem nächsten, hier im April n. J. stattfindenden Anatomencongress zur Demonstration gebracht werden mögen.

Kiel, 18. November 1897. 